\title{
Influence of average cavity dispersion and spectral bandwidth on passively harmonic mode locked L-band Er-doped fiber laser
}

\author{
Qianqian Huang, Chuanhang Zou, Tianxing Wang, Mohammed Al Araimi, Aleksey Rozhin, and \\ Chengbo Mou
}

\begin{abstract}
We systematically study the impact of average cavity dispersion and pulse spectral bandwidth on harmonic mode locking in the long-wavelength band from an Er-doped fiber laser using carbon nanotubes polyvinyl alcohol (CNTs-PVA) film mode locker. By carrying out pulse energy management through optimizing the average cavity dispersion and optical spectral bandwidth, $2.08 \mathrm{GHz}$ repetition rate corresponding to $201^{\text {st }}$ harmonic with $36.5 \mathrm{~dB}$ side mode suppression ratio (SMSR) can be realized under $205 \mathrm{~mW}$ pump power at $1597.53 \mathrm{~nm}$. Furthermore, we systematically investigate the characteristics of the harmonically mode locked ultrashort optical pulses with different spectral bandwidths versus pump power under fixed cavity dispersion firstly. Key laser parameters have been studied including pumping efficiency, duration, energy, SMSR and time bandwidth product (TBP). Enlightened by the experimental results, a simple law on preliminary and fast judgment of pulses frequency under fixed pump power is proposed, namely, by monitoring the pulse spectral bandwidth. The laser source featuring high repetition rate and L-band operation is greatly sought-after in some specific applications such as modern optical communication system etc.
\end{abstract}

Index Terms — Fiber lasers, laser mode locking, nanomaterials

\section{INTRODUCTION}

$\mathrm{N}$ OWADAYS, ultra-short fiber lasers with favorable features of high repetition rate at L-band operation are revealed as effective light sources, which contribute to some potential applications such as optical frequency metrology [1], ultra-fast spectroscopy [2], high speed optical sampling [3] and biomedical diagnostics [4] and so on. Especially, the laser sources also make a great extent development of modern

Manuscript received XXXXXX. This work was supported by the National Natural Science Foundation of China (NSFC) $(61605107,61505244)$ the open program (Grant No.2017GZKF17) of the State Key Laboratory of Advanced Optical Communication Systems and Networks at Shanghai Jiaotong University, China; The Open Fund of IPOC2017B010 (BUPT); Young Eastern Scholar Program at Shanghai Institutions of Higher Learning (QD2015027); " Young 1000 Talent Plan" Program of China and RAEng and the Leverhulme Trust Senior Research Fellowships (LTSRF1617/13/57).

Q. Huang, C. Zou and C. Mou are with The Key Lab of Specialty Fiber Optics and Optical Access Network, Shanghai Institute for Advanced Communication and Data Science, Joint International Research Laboratory of Specialty Fiber Optics and Advanced Communication, Shanghai University, Shanghai, $200444 \quad$ P. $\quad$ R. China (e-mail: cecilin@i.shu.edu.cn; chuanhangzou@i.shu.edu.cn; corresponding author mouc1@ shu.edu.cn). optical communication system [5]. By general agreement for a soliton mode-locked laser, when the pump power exceeds significantly the energy that a single pulse can withstand, the single pulse will split into several pulses due to the peak power limitation effect and energy quantization effect [6], forming multi-soliton state. The observations of a set of multi-soliton patterns have been widely reported, including soliton molecules [7], soliton rains [8], soliton crystal [9], etc. Furthermore, under a specific circumstance, multiple solitons are self-organized and possess ordered distribution with uniform spacing, well known as harmonic mode locking (HML) [10]. Therefore, it is possible to scale the pulses repetition rate up to several gigahertz with the removal of cavity design barriers induced by extra modulators and limitation of fiber length.

Recent years, the widespread applications of mode-locked fiber laser have arisen research upsurge in academia [11-13]. Saturable absorbers (SAs), considered as one of the most efficient mode-locked methods, play a pivotal role in the achievement of ultra-short pulses, which mainly consist of artificial SAs and physical SAs. The artificial SAs are represented by nonlinear polarization rotation (NPR) [14] and nonlinear optical loop mirror (NOLM) [15], while the physical SAs include semiconductor saturable absorber mirror (SESEAM) [16], carbon nanotubes (CNTs) [17] and some cross-sectional novel 2D materials, for instance, graphene [18], black phosphorus [19], topological insulator (TI) [20], and MXene [21]. Interestingly, most of the SAs are also applicable for HML [22-30], which have been under wide exploration. Recently, we also have shown that CNTs polymer film can be considered as an effective SA to realize high repetition rate via

T. Wang is with Key Laboratory of Materials for High Power Laser, Shanghai Institute of Optics and Fine Mechanics, Chinese Academy of Sciences, Shanghai 201800, China (e-mail: wangtianxing@ siom.ac.cn)

M. AlAraimi is now with Higher College of Technology, Al-Khuwair, PO BOX 74, Postal Code 133, Sultanate of Oman (email: mohammed.alaraimi@ hct.edu.om)

A. Rozhin is with Aston Institute of Photonic Technologies (AIPT), Aston University, Birmingham, B4 7ET, United Kingdom (e-mail: a.rozhin@ aston.ac.uk ).

Color versions of one or more of the figures in this paper are available online at http://ieeexplore.ieee.org

Digital Object Identifier xxxxxxxxxxx 
HML [31]. Regarding the distinct advantages of mature manufacture, convenient implementation as well as high flexibility, it is attractive to explore HML using CNTs polymer film.

It is well known that in HML fiber laser, the harmonic order increases along with the elevation of pump power. Therefore, both watts level pumping power and double-cladding gain fiber configurations are intended to be applied in order to generate several gigahertz repetition rate pulses. This complicates the design of the laser system and remains a challenge for widely used applications [32]-[34]. In recent years, some studies have shown great progress on lowering the soliton energy to achieve higher harmonic order to resolve the problem. In 2012, Jun et al experimentally demonstrated the implications of average cavity dispersion and spectral bandwidth on pulse energy [35]. By effectively engineering the pulse energy, $4.9 \mathrm{GHz}$ repetition rate pulses were obtained under $400 \mathrm{~mW}$ pump power in Erdoped laser using evanescent field interaction CNT type [36]. Similarly, Tao et al reported the generation of $22.13 \mathrm{GHz}$ pulses from NPR-based fiber laser by adjusting the average cavity dispersion and average nonlinearity [37]. Those experimental reports have demonstrated that to a certain extent, lower average dispersion and narrower spectral bandwidth are more favorable to decrease the single pulse energy, which results in the intensification of pulse splitting when laser works in C-band region. Nevertheless, considering the widespread applications of high repetition rate fiber lasers in L-band region, it would also be particularly necessary to know whether the key parameters - average cavity dispersion and spectral bandwidth - play the same role when influencing properties of HML. In addition, the aforementioned reports haven't clarified clearly that the cavity dispersion will be in a position to greatly influence the pulse spectral bandwidth.

Here for the first time, we have achieved 2.08 GHz HML at L-band of Er-doped fiber laser mode locked by CNTs-PVA film through a systematic investigation of how the two parameters - the average cavity dispersion and the spectral bandwidth - affect the harmonic order in the L-band. It is found that the pulse energy is eventually dependent on the product of average dispersion value and spectral bandwidth (DBP) when other cavity parameters remain similar. Therefore to some extent, smaller average cavity dispersion and narrower spectral bandwidth are advantageous to pulse splitting in agreement with the regularities enforceable in C-band [36]. Moreover, it is found that minimizing the average cavity dispersion is not an optimal condition to achieve high harmonic order. This is because when the cavity dispersion is decreased to a turning point, the spectral bandwidth will raise dramatically instead. This will increase the product value of the two parameters leading to higher pulse energy. Therefore, it is particularly important to balance the underlying relation between the two factors. Eventually, $2.08 \mathrm{GHz}$ repetition rate pulses with 4.83 $\mathrm{nm} 3 \mathrm{~dB}$ bandwidth at $201^{\text {st }}$ harmonic are realized under 205 $\mathrm{mW}$ pump power. The corresponding pulse energy is $3.44 \mathrm{pJ}$, which is the lowest generated from CNTs-based HML fiber laser, implying effective pulse energy management. Furthermore, the pulses center at $1597.53 \mathrm{~nm}$ with $36.5 \mathrm{~dB}$
SMSR, indicating stable operation in L-band region. The laser source exhibiting fascinating aspects of several gigahertz frequency and L-band operation simultaneously makes great effort to some applications such as optical communication system. Additionally, for the first time, we carried out the systematic investigation on the performances of the pulses with different spectral bandwidth versus pump power under a fixed cavity dispersion including pumping efficiency, duration, energy, SMSR and TBP. According to the experimental results, we also reveal a simple way to judge empirically the frequency of the pulses under a fixed pump power, namely, monitoring the pulse spectral bandwidth. Our work can be served as a reference guide for designing a high-order passively HML fiber laser.

\section{The Soliton Area THEOREM}

The pulse energy engineering can be traced back to the soliton area theorem [38], which is a key foundation for the possibility to expand repetition rate through HML. From the soliton area theorem, the soliton area is constrained to the product of $A_{0}$ and $\tau$, which represent the pulse peak amplitude and pulse width at $1 / \mathrm{e}$ :

$$
\text { soliton area }=A_{0} \tau=\sqrt{\frac{2|D|}{\delta}} .
$$

$D$ and $\delta$ are as defined by the following (2) and (3).

$$
\begin{aligned}
D & =\frac{1}{2} \beta_{2, \text { ave }} \cdot L, \\
\delta & =\gamma_{\text {ave }} \cdot L,
\end{aligned}
$$

where $\beta_{2, a v e}$ and $L$ stand for the average cavity dispersion and cavity length, while $\gamma_{\text {ave }}$ is the average nonlinearity parameter. The single pulse energy $\mathrm{W}$ can be specified by

Considering $(1) \sim(4)$, we can get:

$$
\mathrm{W}=2\left|A_{0}\right|^{2} \tau
$$

$$
\mathrm{W}=2\left|A_{0}\right|^{2} \tau=\frac{\left|\beta_{2, \text { ave }}\right|}{\gamma_{\text {ave }}} \cdot \frac{1}{\tau} .
$$

Considering $T_{F W H M}=1.763 \tau$ when the pulse has a hyperbolic secant shape, the pulse energy is then stated by

$$
\mathrm{W}=\frac{\left|\beta_{2, \text { ave }}\right|}{\gamma_{\text {ave }}} \cdot \frac{1.763}{T_{F W H M}},
$$

where $T_{F W H M}$ is the pulse duration at full-width half maximum (FWHM).

As we known, the time bandwidth product (TBP) is calculated as follows:

$$
\mathrm{TBP}=\frac{T_{F W H M} \cdot \Delta \lambda \cdot \mathrm{c}}{\lambda_{c}{ }^{2}} .
$$

Here $\mathrm{c}, \Delta \lambda$ and $\lambda_{c}$ are light speed, $3 \mathrm{~dB}$ bandwidth of optical spectrum and center wavelength respectively. Then, the pulse energy $W$ can be expressed by the equation:

$$
\mathrm{W}=\frac{\left|\beta_{2, \text { ave }}\right|}{\gamma_{\text {ave }}} \cdot \frac{1.763}{T_{F W H M}}=1.763 \times \frac{\left|\beta_{2, \text { ave }}\right|}{\gamma_{\text {ave }}} \cdot \frac{\Delta \lambda \cdot \mathrm{c}}{\mathrm{TBP} \cdot \lambda_{c}{ }^{2}} .
$$

From (8), it is apparent that the pulse energy is determined by the product of $D B P=\left|\beta_{2, \text { ave }}\right| \cdot \Delta \lambda$ when other parameters are similar such as $\gamma_{\text {ave }}$, TBP and $\lambda_{c}$. In general, smaller average dispersion and narrower spectral bandwidth leading to smaller DBP value thus are more favorable in a position to lower the pulse energy with a consequence of possible higher harmonic order. This has been proved in the laser working at C-band regime [36]. However, since many HML fiber lasers expand their working wavelength range to L-band, a question 
spontaneously emerged as to whether the regularity can still take effect in L-band region, which will be expressed detailly in the following parts. Also, it has been known that the spectral bandwidth is susceptible to the cavity dispersion [37], [39], [40]. When the net cavity dispersion reduces to near zero, the spectral bandwidth will increase dramatically. As a result, DBP will become larger instead and the pulse energy will rise correspondingly. Therefore, a delicate balance between the two factors is required to optimize the DBP.

\section{FABRICATION AND CHARACTERRISTICS OF CNTS-PVA FILM}

\section{A. Fabrication of CNTS-PVA Film}

The preparation of CNTs-PVA film can be summarized in four steps, which is similar to that in Ref [41]. Firstly, we put 2 $\mathrm{mg}$ single walled CNTs (HiPco, Unidym) into $10 \mathrm{ml}$ deionized water and added sodium dodecylbenzene sulfonate (SigmaAldrich) surfactant as dispersion agent. Then, the mixture is subjected to sonication introduced by an ultrasonic apparatus (Nanoruptor, Diagenode) under the conditions of $200 \mathrm{~W}$ power and $20 \mathrm{kHz}$ frequency. This provides a condition for uniform dispersion of CNTs particles into the aqueous solution. After that, ultracentrifugation is carried out for an hour with 25000 RPM for the purpose of eliminating impurities and large CNT bundles. Afterwards, the PVA powders are decanted into the prepared suspension and then poured into a Petri dish, which is finally placed in a desiccator to evaporate water for several days. As a result, the CNTs-PVA film is formed. After cutting the film into $1 \times 1 \mathrm{~mm}$ piece, we can get a practical SA by sandwiching it between two fiber connectors directly.

\section{B. Characteristics of CNTS-PVA Film}

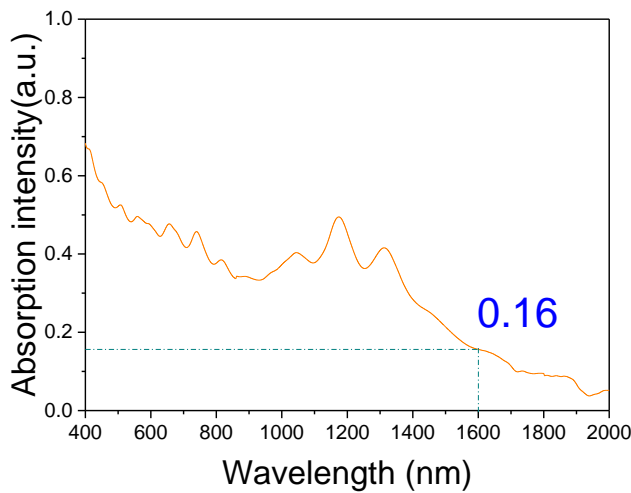

(a)

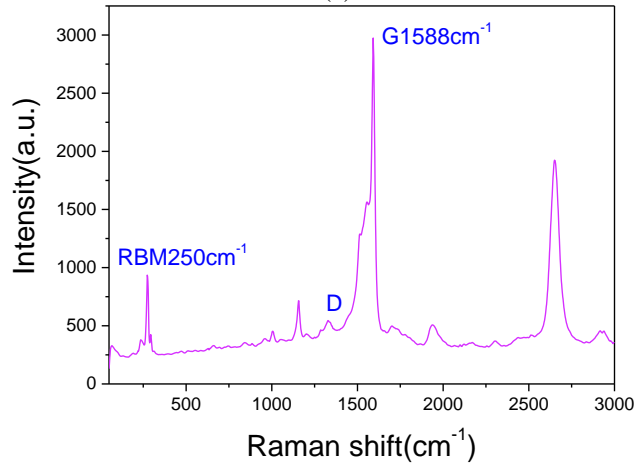

(b)

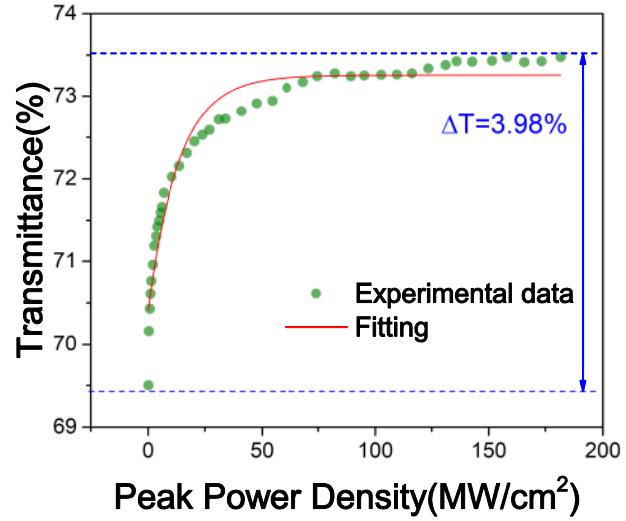

(c)

Fig. 1. The characteristics of the CNTs-PVA film: (a) The absorption spectrum; (b) The Raman spectrum meaured by $532 \mathrm{~nm}$ pumping light; (c) The measured nonlinear transmission.

The linear optical absorption spectrum and Raman spectrum of CNT-PVA film are shown in Fig. 1(a) and Fig. 1(b). The absorption spectrum has a number of absorption bands between $400 \mathrm{~nm}$ to $2000 \mathrm{~nm}$, and the absorption intensity at $1600 \mathrm{~nm}$ approaches 0.16. Furthermore, from Fig. 1(b), we can see that two pronounced peaks are radial breathing mode (RBM) and the $\mathrm{G}$ mode respectively. Notably, based on $250 \mathrm{~cm}^{-1} \mathrm{RBM}$, the mean diameter is estimated to be $0.88 \mathrm{~nm}$. Both Raman and absorption spectra confirm that the CNTs are single walled. Also, a home-made laser delivering stable mode locked pulses at $1595 \mathrm{~nm}$ with $19 \mathrm{~mW}$ output power is applied to measure the nonlinear transmission illustrated in Fig. 1(c), giving the modulation depth of $3.98 \%$. Taking advantages of such high modulation depth and 0.16 absorption intensity at $1600 \mathrm{~nm}$, it is expected that the CNTs-PVA film can be deemed as a practical mode locker at L-band.

\section{EXPERIMENTAL SET-UP}

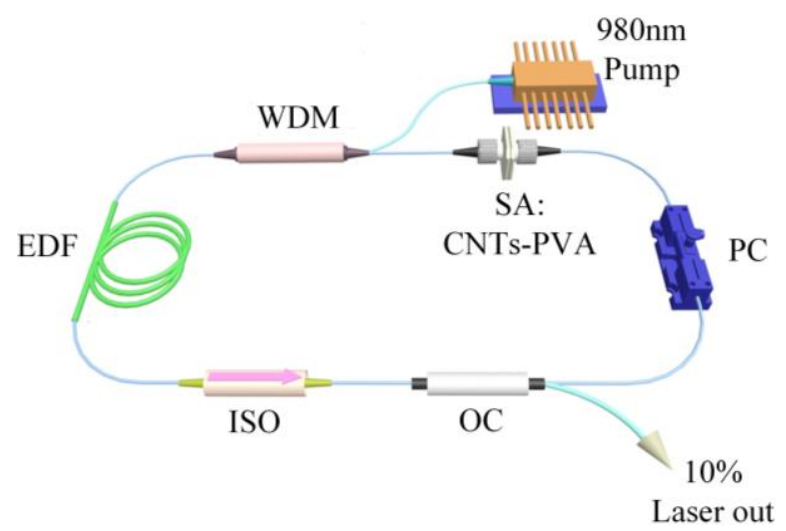

Fig. 2. The schematic configuration of L-band Er-doped fiber laser using CNTs-PVA film.

The configuration of the demonstrated fiber laser with CNTs-PVA mode-locker is shown in Fig. 2. The dispersion managed loop cavity is organized by three types of fibers: Erdoped fiber (EDF Er30-4/125 from Liekki), OFS 980 fiber and single mode fiber (SMF), whose group velocity delays (GVDs) 
are $+14.45 \mathrm{ps}^{2} / \mathrm{km},+4.5 \mathrm{ps}^{2} / \mathrm{km}$ and $-22.8 \mathrm{ps}^{2} / \mathrm{km}$, respectively. The EDF used is $8.18 \mathrm{~m}$ with slightly smaller mode field diameter of $6.5 \pm 0.5 \mu \mathrm{m}$, which cannot only lead to L-band operation but also possess high nonlinearity. It is considered that the L-band emission comes from in-band absorption due to the comparatively long length EDF with highly-doped concentration [42]. Since only SMF has negative dispersion in the cavity, it can be utilized to compensate the cavity dispersion. Therefore, we can get various average cavity dispersion just by decreasing the length of SMF. A benchtop laser (OV LINK, Wuhan, China) operating at $980 \mathrm{~nm}$ is adopted to launch pump light into the cavity through a fused taper type wavelengthdivision multiplexer (WDM). The clockwise propagation of light results from the use of an isolator (ISO). A fused output coupler spliced after ISO permits $10 \%$ light out of the cavity. The mode-locker device is formed by sandwiching CNTs-PVA film between two fiber connectors. A polarization controller (PC) is employed to optimize the polarization state of the laser. The obtained pulses are detected by several experimental instruments including an optical spectrum analyzer (OSA, Yokogawa AQ6370C), an $8 \mathrm{GHz}$ oscilloscope (OSC, KEYSIGHT DSO90804A), a radio frequency (RF) spectrum analyzer (SIGLENT, SSA 3032X), a $12.5 \mathrm{GHz}$ photo-detector (PD, Newport 818- BB-51F) and an autocorrelator (FEMTOCHROME, FR-103WS).

\section{INFLUENCE OF AVERAGE CAVITY DISPERSION AND SPECTRAL BANDWIDTH ON HML}

\section{A. Influence of Average Cavity Dispersion on $H M L$}

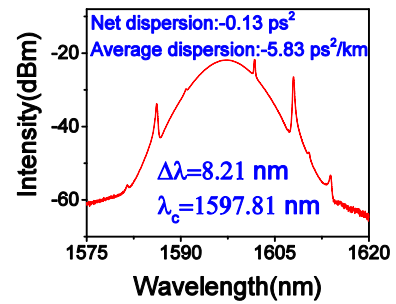

(a)

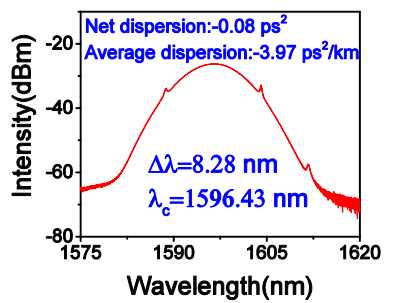

(b)

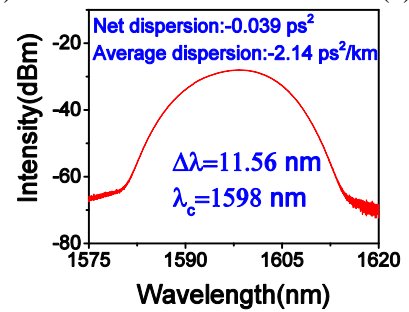

(c)

Fig. 3. The representative optical spectra with different dispersion: (a) Optical spectrum of conventional soliton under cavity dispersion of $-0.13 \mathrm{ps}^{2}$; (b) Optical spectrum of conventional soliton under cavity dispersion of $-0.08 \mathrm{ps}^{2}$; (c) Optical spectrum of stretched-pulse under cavity dispersion of $-0.039 \mathrm{ps}^{2}$.

In order to explore the impact of average cavity dispersion on HML, we deliberately change the length of intracavity SMF which results in the variation of average dispersion value. In the experiment, $\beta_{2, \text { ave }}$ varies from $-5.83 \mathrm{ps}^{2} / \mathrm{km}$ to $-3.97 \mathrm{ps}^{2} / \mathrm{km}$ and further down to $-2.14 \mathrm{ps}^{2} / \mathrm{km}$, when the length of intracavity SMF is tailored from $1142 \mathrm{~cm}$ to $921 \mathrm{~cm}$ and to 742 $\mathrm{cm}$. The total cavity lengths are $22.35 \mathrm{~m}, 20.14 \mathrm{~m}$ and $18.35 \mathrm{~m}$, corresponding to fundamental repetition rates of $9.32 \mathrm{MHz}$, $10.34 \mathrm{MHz}$ and $11.35 \mathrm{MHz}$, respectively. Fig 3 presents the representative optical spectra at different average cavity dispersion which all clearly locate at L-band region. In the cases of $\beta_{2, a v e}=-5.83 \mathrm{ps}^{2} / \mathrm{km}$ and $\beta_{2, \text { ave }}=-3.97 \mathrm{ps}^{2} / \mathrm{km}$, the clear existence of Kelly sidebands features typical characteristics of optical soliton, while the optical spectrum in the situation of $\beta_{2, \text { ave }}=-2.14 \mathrm{ps}^{2} / \mathrm{km}$ exhibits Gaussian-form profile without Kelly sidebands, indicating the generation of stretched-pulse [43]. The frequency and harmonic order obtained at different $\beta_{2, \text { ave }}$ are plotted in Fig. 4 under the pump power of $190 \mathrm{~mW}$. Along with the decreasing of $\beta_{2, \text { ave }}$, the frequency rises from $456.76 \mathrm{MHz}$ to $763.15 \mathrm{MHz}$ and 1224.97 $\mathrm{MHz}$, corresponding to the elevation of harmonic order from $49^{\text {th }}$ to $74^{\text {th }}$ and $108^{\text {th }}$. Furthermore, for the purpose of better demonstrating the influence of $\beta_{2, \text { ave }}$ on HML, Fig. 5 summarizes the repetition frequency as a function of pump power at $\beta_{2, \text { ave }}=-5.83 \mathrm{ps}^{2} / \mathrm{km},-3.97 \mathrm{ps}^{2} / \mathrm{km}$ and $-2.14 \mathrm{ps}^{2} / \mathrm{km}$, respectively. It can be clearly seen that all pumping efficiency slopes show linear tendency, which is one of the typical characteristics of harmonic mode locked fiber laser [26, 29-31]. In general, the smaller pulse energy can lead to larger pump harmonic efficiency. Therefore, the smaller average cavity dispersion leads to lower pulse energy (10 15 $\mathrm{pJ}, 8 \sim 11 \mathrm{pJ}$, 3.6 4.1 pJ) and larger pump harmonic efficiency (3.78 $\mathrm{MHz} / \mathrm{mW}, 8.91 \mathrm{MHz} / \mathrm{mW}, 8.99 \mathrm{MHz} / \mathrm{mW}$ ), which coincides with the behavior of C-band operation [36], [37]. However, it is clear that the orange one is only a little bit larger than the blue one. It is postulated that the different cavity dispersion and different intracavity polarization state may lead to different conversion efficiency of pump light, thus influencing the pump harmonic efficiency. We also calculate the average DBP values for the cases of $\beta_{2, a v e}=-5.83 \mathrm{ps}^{2} / \mathrm{km},-3.97 \mathrm{ps}^{2} / \mathrm{km}$ and -2.14 $\mathrm{ps}^{2} / \mathrm{km}$, which are $40.85,32.39$ and 25.78 respectively. It is clear that smaller DBP value results in lower pulse energy, agreeing well with the expression in part II. Furthermore, one may notice that when the net dispersion deceases near zero, i.e. $\beta_{2, a v e}=-2.14 \mathrm{ps}^{2} / \mathrm{km}$, the pulse spectrum spreads broader due to entering the stretched-pulse scheme. Nevertheless, the DBP value is still smaller than those of the rest chosen cavity average dispersion conditions. This means that the stretching degree of the spectral bandwidth here only have a marginal effect on the value of DBP, which can be neglected during the abovementioned experiments. As described so far, we can conclude that in L-band region, decreasing the average dispersion of cavity can deduce DBP value to lower the pulse energy, giving rise to the achievement of an appreciable repetition rate. 


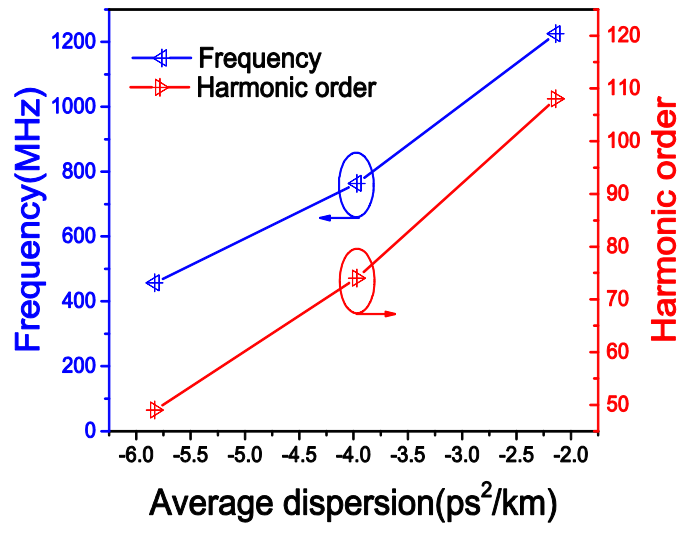

Fig. 4. The frequency and harmonic order versus average dispersion under 190 $\mathrm{mW}$ pump power.

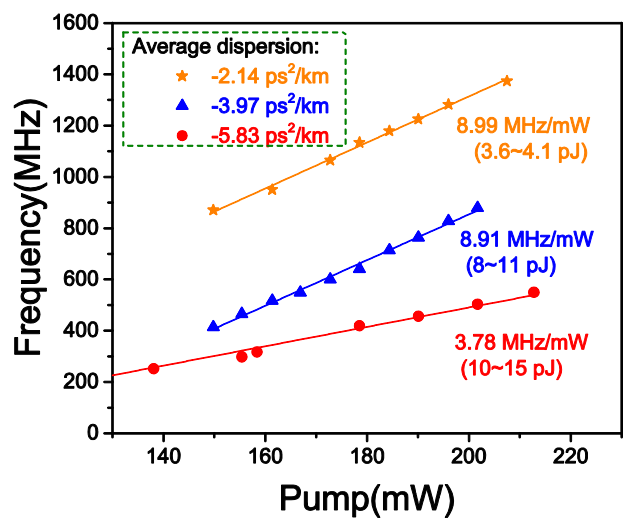

Fig. 5. The variations of frequency with different average dispersion as a function of pump power (i.e. $-2.14 \mathrm{ps}^{2} / \mathrm{km}$ [orange star], $-3.97 \mathrm{ps}^{2} / \mathrm{km}$ [blue triangle], and $-5.83 \mathrm{ps}^{2} / \mathrm{km}$ [red dot]).

\section{B. Influence of Spectral Bandwidth on HML}

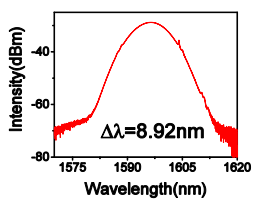

(a)

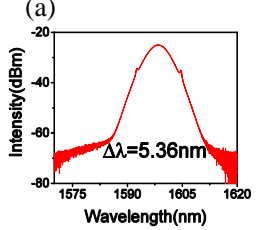

(d)

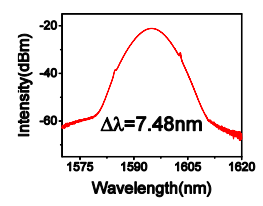

(b)

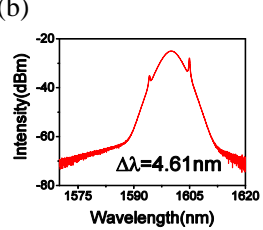

(e)

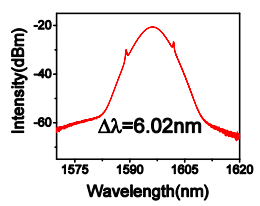

(c)
Fig. 6. The typical recorded pulse spectra at different spectral bandwidth region under a fixed average dispersion of $-3.97 \mathrm{ps}^{2} / \mathrm{km}$ : (a) $8.1 \sim 9.6 \mathrm{~nm}$. (b) $7.1 \sim 7.7$ nm. (c) $5.6 \sim 6.3 \mathrm{~nm}$. (d) $5 \sim 5.55 \mathrm{~nm}$. (e) $4.4 \sim 4.96 \mathrm{~nm}$.

In the aforementioned experiment, it is found that the pulse spectral bandwidth can be changed by simply adjusting the rotation of PC. Although there is no polarizing component in the cavity, not only the fused taper type components including OC and WDM but also EDF exhibiting polarization dependent gain [44] caused by erbium ion anisotropy [45] can induce polarization dependent characteristic. Combined with large intracavity birefringence due to the relatively long cavity length, the artificial cavity birefringence filter is formed [46-47]. It is considered that the spectral bandwidth tunability is rooted in the different effective filtering bandwidth when PC is adjusted [48]. The tuning ranges of spectral bandwidth are $6 \sim 8.5 \mathrm{~nm}$, 4.4 9.6 $\mathrm{nm}, 11 \sim 13 \mathrm{~nm}$ respectively under $\beta_{2, \text { ave }}=-5.83 \mathrm{ps}^{2} / \mathrm{km}$, $-3.97 \mathrm{ps}^{2} / \mathrm{km}$ and $-2.14 \mathrm{ps}^{2} / \mathrm{km}$. We can clearly see that in the case of $\beta_{2, \text { ave }}=-3.97 \mathrm{ps}^{2} / \mathrm{km}$, the tuning range of spectral bandwidth is the largest among the three chosen cavity average dispersion conditions. So we investigate the influence of spectral bandwidth on HML at the fixed average cavity dispersions of $\beta_{2, \text { ave }}=-3.97 \mathrm{ps}^{2} / \mathrm{km}$. Under fixed $\beta_{2, \text { ave }}$, by merely tuning the PC, we can obtain five distinct HML modes identified by obvious different spectral bandwidths regions (8.1 9.6 nm, 7.1 7.7 nm, 5.6 6.3 nm, 5 5.55 nm, 4.4 4.96 $\mathrm{nm})$. This is depicted in Fig. 6. From the picture, we can see that the soliton operations are characterized by the existence of Kelly sidebands in all spectra. Also, it comes to our attention that the variation of pulse spectral bandwidth is responsible for the change of the repetition rate, as shown in Fig. 7(a). Generally, the narrower spectral bandwidth the pulse exhibits, the higher repetition rate the pulses are, which is still similar to the operation in C-band [36]. Correspondingly, with the decreasing of spectral bandwidth, the pulse energy changes from $9 \sim 10.8 \mathrm{pJ}$ to $6 \sim 7.5 \mathrm{pJ}, 4.6 \sim 6 \mathrm{pJ}, 3.8 \sim 4.5 \mathrm{pJ}$ and $3.4 \sim 3.8$ pJ, as depicted in Fig. 7(b) and the average DBP values are 33.8, $29.1,23.2,21.18$ and 19.08 by calculation. We can see that the lower DBP value leads to lower pulse energy, which is consistent with the results described in part II. Particularly, the pump harmonic efficiency is increased with the decreasing of optical spectral bandwidth (except for the green one). However, the pump harmonic efficiency of the green one is smaller than we expected. It is postulated that in the case, the intracavity polarization state may lead to smaller conversion efficiency of pump light [44]. Worthy of mentioning, at the fixed average dispersion of $-3.97 \mathrm{ps}^{2} / \mathrm{km}$, the efficiency of the largest pumping slope reaches $13 \mathrm{MHz} / \mathrm{mW}$ and the highest frequency is $2.08 \mathrm{GHz}$. Both of them are higher than those (pump harmonic efficiency of $8.99 \mathrm{MHz} / \mathrm{mW}$, highest frequency of $1.374 \mathrm{GHz})$ in the case of $\beta_{2, \text { ave }}=-2.14 \mathrm{ps}^{2} / \mathrm{km}$ and $\Delta \lambda=$ $11 \sim 13 \mathrm{~nm}$, as shown in Fig. 5. The pulse energies are $3.44 \mathrm{pJ}$ and $3.6 \mathrm{pJ}$ and the calculated DBP are 19.2 and 25.7 respectively in the cases of $2.08 \mathrm{GHz}$ and $1.374 \mathrm{GHz}$. Thereby, considering the influence of average dispersion on spectral bandwidth, we optimize the relationship between them for the first time to obtain the smallest DBP value. As a result, the harmonic order we obtain is sufficiently improved.

Furthermore, systematic investigation on other properties of HML with different spectral bandwidth is carried out for the first time. As we know, the pivotal characteristic of stability in HML operation is expressed by the value of SMSR. From Fig. 7(c), we can clearly see that the narrower spectral bandwidth with higher repetition rate shows lower SMSR value. We conjecture it from the stronger competition between fundamental and harmonic frequencies when the harmonic 
order is higher [27], [49]. Here, we would like to emphasize that the lowest SMSR in the case of $\Delta \lambda=4.4 \sim 4.96 \mathrm{~nm}$ is still up to $35 \mathrm{~dB}$, manifesting excellent stability. In addition, Fig 7(d) plots the resulting pulse duration in the cases of different spectral bandwidth versus pump power, where the narrower spectral bandwidth shows larger pulse duration which is consistent with Fourier transform. It should be guaranteed that the pulse TBP possesses slight variation in the range of 0.65 0.9, which manifesting slight pulse chirp, as depicted in Fig. 7(e). Although the soliton area theorem only applies to the conventional soliton, which contains no chirp generally. Both J.H. Eberly and William H. Renninger have demonstrated that pulse chirp can merely change the pulse phase without affecting the pulse energy [50], [51]. Therefore, the soliton area theorem is still applicable to our case.

The experimental observation is well consistent with the analysis in Part II. And stimulated by the experimental results, we have developed a simple method to roughly identify the repetition rate by monitoring pulse spectral bandwidth, which can be performed to find the approximate scope of desired frequency quickly. Worthy of mentioning, our laser always works at L-band region throughout the experiment. Meanwhile, steady operation can be maintained for at least several hours when stable HML occurs. Moreover, in our work, the CNTsbased SA can work for several weeks steadily if the pump power is controlled well.

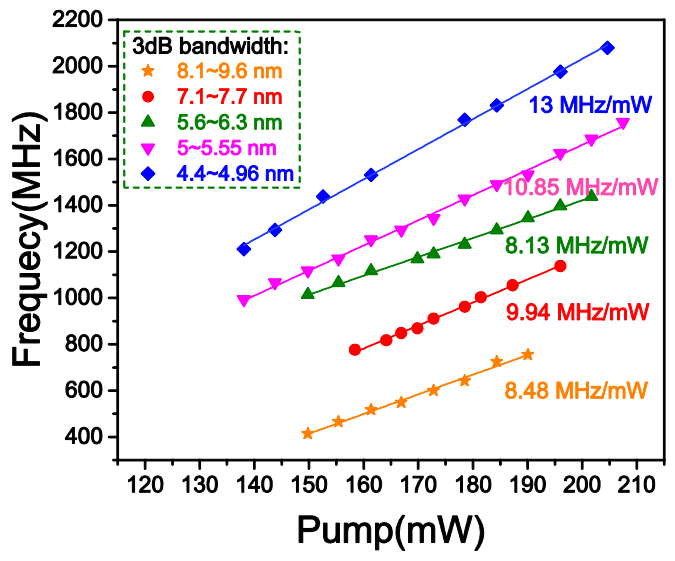

(a)

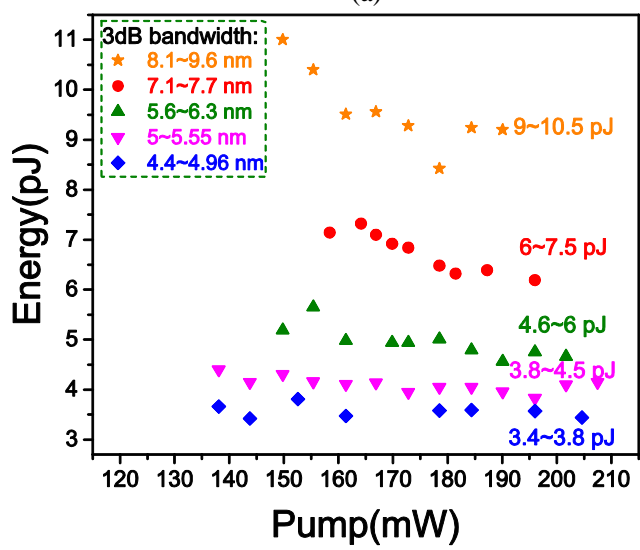

(b)

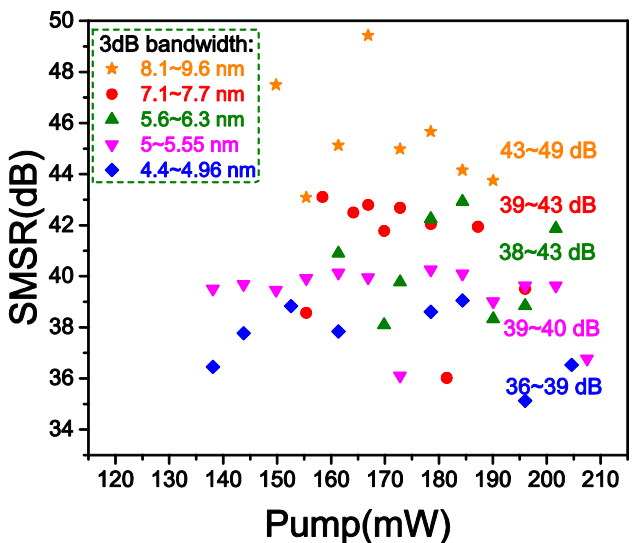

(c)

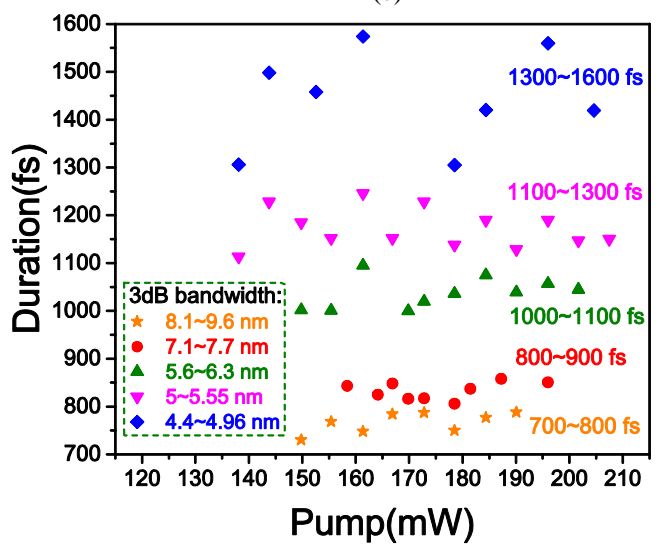

(d)

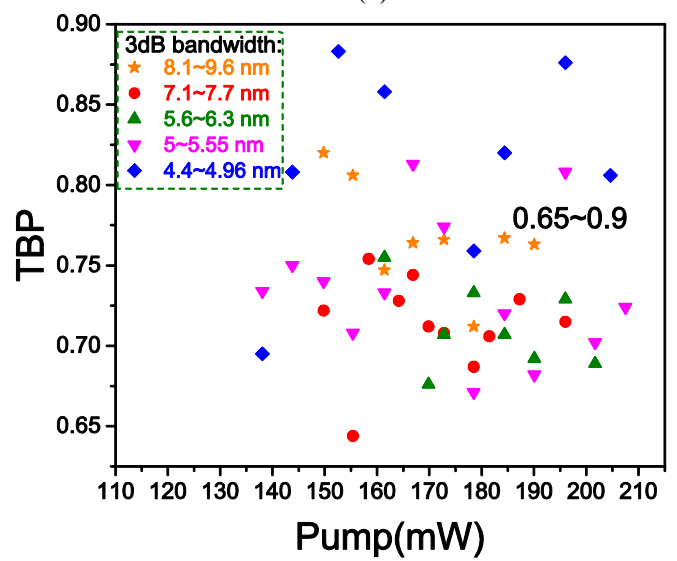

(e)

Fig. 7. The characteristics of HML pulses at different spectral bandwidth region against pump power change (a) repetition frequency; (b) pulse energy; (c) SMSR value; (d) pulse duration; (e) TBP. (i.e. 8.1 9.6 nm [orange star], $7.1 \sim 7.7 \mathrm{~nm}$ [red dot], $5.6 \sim 6.3 \mathrm{~nm}$ [olive triangle], $5 \sim 5.55 \mathrm{~nm}$ [pink triangle], and 4.4 4.96 nm [blue diamond])

\section{CHARACTERISTICS OF 2GHZ PULSES}

By carefully collaborative optimization of average dispersion and spectral bandwidth to make DBP small enough, the laser can yield $2.08 \mathrm{GHz}$ pulses at $201^{\text {st }}$ harmonic which is the highest record in our experiment under $205 \mathrm{~mW}$ pump power. The well-characterized pulse train with equi-distant spacing and similar amplitude is visualized in Fig. 8(a). The measured pulse spacing is $0.48 \mathrm{~ns}$ in consent with the pulse frequency. 
The corresponding optical spectrum is presented in Fig. 8(b), whose $3 \mathrm{~dB}$ bandwidth and center wavelength are $4.83 \mathrm{~nm}$ and $1597.53 \mathrm{~nm}$, respectively, which means that the laser works in L-band region. Fig 8(c) shows the measured RF spectra in 3.2 $\mathrm{GHz}$ span, where the maximum peak in the position of 2.08 $\mathrm{GHz}$ gives a further verification of pulse repetition rate. And the SMSR is $36.5 \mathrm{~dB}$ while signal-to-noise ratio is $56.6 \mathrm{~dB}$, manifesting steady operation. Fig 8(d) displays the measured autocorrelation trace. When the pulse shape is set to secant hyperbolic profile, the pulse duration is estimated to $1.42 \mathrm{ps}$. Therefore, the TBP is 0.806 indicating slightly pulse chirp. Furthermore, accounting for the output power of $7.15 \mathrm{~mW}$, the pulse energy is about $3.44 \mathrm{pJ}$, which is the lowest [35] generated from CNTs-based laser confirming the effective of balancing the relationship between average dispersion and spectral bandwidth.

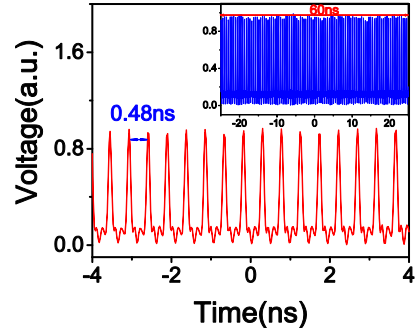

(a)

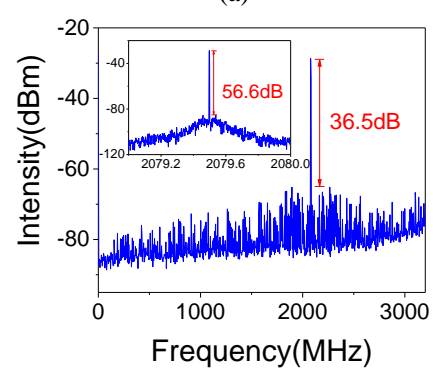

(c)

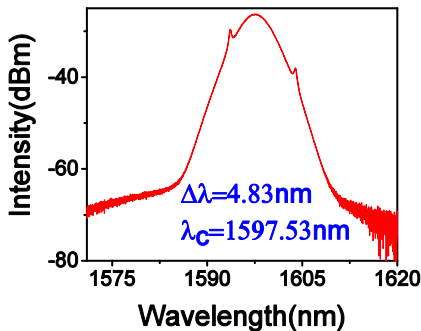

(b)

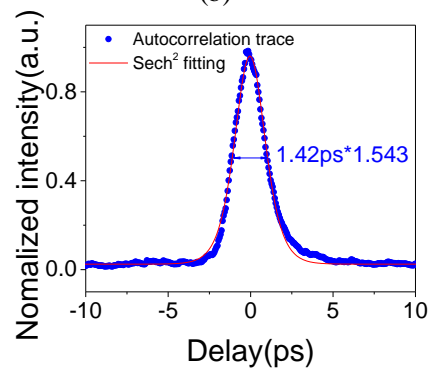

(d)
Fig. 8 . The characteristics of $2.08 \mathrm{GHz}$ pulses under $395 \mathrm{~mW}$ pump power: (a) The time domain waveform visualized in oscilloscope; (b) The measured optical spectrum; (c) The detected RF spectrum in $3.2 \mathrm{GHz}$ span with resolution of $1 \mathrm{MHz}$, and inset is RF spectrum measured with $1 \mathrm{MHz}$ span and $1 \mathrm{kHz}$ resolution bandwidth; (d) The measured autocorrelation trace.

\section{CONCLUSION}

In conclusion, we experimentally identified that in the L-band region, the pulse energy is eventually determined by the product of the cavity average dispersion and pulse spectral bandwidth when other cavity parameters are similar. To some extent, smaller average dispersion and narrower spectral bandwidth are essentially required for depressing pulse energy substantially, which is similar to the C-band described in Ref [36]. We also find out that there is an optimal value of average dispersion due to the influence on spectral bandwidth. By controlling the balance between the two parameters carefully to make the product value smaller, our laser allows the generation of $2.08 \mathrm{GHz}$ pulses at $201^{\text {st }}$ harmonic with $13 \mathrm{MHz} / \mathrm{mW}$ pump harmonic efficiency. The outstanding stability is attested by the SMSR value of $36.5 \mathrm{~dB}$. Furthermore, we systematically investigate the influence of spectral bandwidth on the performances of HML under a fixed dispersion in terms of pumping efficiency, energy, SMSR, duration and TBP for the first time. Enlightened by the experimental observation, a simple approach based on monitoring spectral bandwidth is presented here, which is beneficial for the preliminary judgment of the wanted repetition rate. Additionally, our study implies guidelines in the construction of HML fiber laser. Specifically, taking into account the effect of cavity nonlinearity on pulse energy according to equation (8) and Ref [37], our future work will focus on engineering the cavity nonlinearity to scale the repetition rate further.

\section{REFERENCES}

[1] Jones R J and Diels J-C, "Stabilization of femtosecond lasers for optical frequency metrology and direct optical to radio frequency synthesis," Phys. Rev. Lett., vol. 86, no. 1, pp. 3288 Apr. 2001.

[2] Marshall J, Stewart G, and Whitenett G, "Design of a tunable L-band multiwavelength laser system for application to gas spectroscopy," Meas. Sci. Technol., vol. 17, no. 2, pp. 1023 Apr. 2006.

[3] Schlager J B, Hale P D, and Franzen D L, "High-sensitivity optical sampling using an erbium-doped fiber laser strobe," Microwave Opt. Technol. Lett., vol. 6, no. 4, pp. 835-837 Dec. 1993.

[4] Okhotnikov O, Grudinin A, and Pessa M, "Ultra-fast fibre laser systems based on SESAM technology: new horizons and applications," New J. Phys., vol. 6, no. 3, pp. 211-218 Oct. 2004.

[5] Srivastava A, Radic S, Wolf C, Centanni J, Sulhoff J, Kantor K, and Sun Y, "Ultradense WDM transmission in L-band," IEEE Photonics Technol. Lett., vol. 12, no. 13, pp. 1570-1572 Nov. 2000.

[6] Thoen E, Koontz E, Joschko M, Langlois P, Schibli T, Kärtner F, Ippen E, and Kolodziejski L, "Two-photon absorption in semiconductor saturable absorber mirrors," Appl. Phys. Lett., vol. 74, no. 5, pp. 3927-3929 May. 1999.

[7] Lin S-F, Lin Y-H, Cheng C-H, Chi Y-C, and Lin G-R, "Stability and chirp of tightly bunched solitons from nonlinear polarization rotation mode-locked erbium-doped fiber lasers," J. Lightwave Technol., vol. 34, no. 7, pp. 51185128 Month 2016.

[8] Wang Z, He R, Liu Y-g, Zhang H, Han S, Li H, Wang G, Yang G, and Wang $Z$, "Generation of trapezoidal envelope pulses and soliton rains from passively mode-locked fiber laser with $\mathrm{MoS}_{2}$ saturable absorber on microfiber," Appl. Phys. Express, vol. 11, no. 30, pp. 072504 Jun. 2018.

[9] Dikandé A M, "Pulse train uniformity and nonlinear dynamics of soliton crystals in mode-locked fiber ring lasers," JOSA B, vol. 34, no. 8, pp. 66-75 Dec. 2017.

[10] Mou C, Arif R, Rozhin A, and Turitsyn S, "Passively harmonic mode locked erbium doped fiber soliton laser with carbon nanotubes based saturable absorber," Opt. Mater. Express., vol. 2, no. 29, pp. 884-890 Jun. 2012.

[11] U. Keller, "Recent developments in compact ultrafast lasers," Nat., vol. 424, no. 6950 , pp. 831-838, Aug. 2003.

[12] B. Oktem, C. Ülgüdür, and F. Ö. Ilday, "Soliton-similariton fibre laser," Nat. Photonics, vol. 4, no. 5, pp. 307-311, May. 2010.

[13] Y. Chen, M. Chang, W. Zhuang, K. Su, K. Huang, and H. Liang, "Generation of sub-terahertz repetition rates from a monolithic self-modelocked laser coupled with an external Fabry-Perot cavity," Laser Photonics Rev., vol. 9, no. 1, pp. 91-97, Oct. 2015.

[14] L. Zhao, D. Tang, X. Wu, and H. Zhang, "Dissipative soliton generation in Yb-fiber laser with an invisible intracavity bandpass filter," Opt. Lett., vol. 35, no. 16, pp. 2756-2758, Aug. 2010.

[15] I. N. Duling, "All-fiber ring soliton laser mode locked with a nonlinear mirror," Opt. Lett., vol. 16, no. 8, pp. 539-541, Apr. 1991.

[16] J. Shi et al., "95 $\mathrm{nJ}$ dispersion-mapped amplifier similariton fiber laser at 8.6 $\mathrm{MHz}$ repetition rate with linear cavity configuration," Opt. Express, vol. 23, no. 14, pp. 18330-18337, Jul. 2015.

[17] S. Y. Set, H. Yaguchi, Y. Tanaka, and M. Jablonski, "Laser mode locking using a saturable absorber incorporating carbon nanotubes," J. Lightwave Technol., vol. 22, no. 1, pp. 51-56, Jan. 2004. 
[18] J. Sotor, I. Pasternak, A. Krajewska, W. Strupinski, and G. Sobon, "Sub-90 fs a stretched-pulse mode-locked fiber laser based on a graphene saturable absorber," Opt. Express, vol. 23, no. 21, pp. 27503-27508, Oct. 2015.

[19] J. Ma et al., "Few-layer black phosphorus based saturable absorber mirror for pulsed solid-state lasers," Opt. Express, vol. 23, no. 17, pp. 22643-22648, 2015.

[20] Y. Chen et al., "Large energy, wavelength widely tunable, topological insulator Q-switched erbium-doped fiber laser," IEEE J. Sel. Top. Quantum Electron. vol. 20, no. 5, pp. 315-322, Sep. 2014.

[21] X. Jiang et al., "Broadband Nonlinear Photonics in Few-Layer MXene Ti3C2Tx (T= F, O, or OH)," Laser Photonics Rev., vol. 12, no. 2, pp. 1700229-1-1700229-10, Nov. 2018.

[22] Grudinin A B, Richardson D J, and Payne D N, "Passive harmonic modelocking of a fibre soliton ring laser," Electron. Lett., vol. 29, no. 28, pp. 1860-1861 Oct. 1993

[23] Sawaguchi C, Sotobayashi H, Chujo W, and Koyamada Y, "Ultrafast walkoff-free nonlinear optical loop mirror by a simplified configuration for 320Gbit/s time-division multiplexing signal demultiplexing," Opt. Lett., vol. 27, no. 14, pp. 1555-1557 Sep. 2002.

[24] Y D, M K, F L, G W, and W K, "Colliding-pulse passive harmonic modelocking in a femtosecond Yb-doped fiber laser with a semiconductor saturable absorber," Opt. Express., vol. 12, no. 15, pp. 3872-3877 Aug. 2004.

[25] Jiang K, Fu S, Shum P, and Lin C, "A Wavelength-Switchable Passively Harmonically Mode-Locked Fiber Laser With Low Pumping Threshold Using Single-Walled Carbon Nanotubes," IEEE Photonics Technol. Lett., vol. 22 , no. 16 , pp. 754-756 Jun. 2010.

[26] Sobon G, Sotor J, and Abramski K M, "Passive harmonic mode-locking in Er-doped fiber laser based on graphene saturable absorber with repetition rates scalable to $2.22 \mathrm{GHz}, "$ Appl. Phys. Lett., vol. 100, no. 18, pp. 3077 3083 Apr. 2012.

[27] Meng Y, Niang A, Guesmi K, Salhi M, and Sanchez F, "1.61 $\mu \mathrm{m}$ high-order passive harmonic mode locking in a fiber laser based on graphene saturable absorber," Opt. Express, vol. 22, no. 9, pp. 29921-29926 Dec. 2014.

[28] Liu M, Luo A-P, Zheng X-W, Zhao N, Liu H, Luo Z-C, Xu W-C, Chen Y, Zhao C-J, and Zhang H, "Microfiber-based highly nonlinear topological insulator photonic device for the formation of versatile multi-soliton patterns in a fiber laser," J. Lightwave Technol., vol. 33, no. 6, pp. 2056-2061 May. 2015.

[29] Luo Z C, Liu M, Liu H, Zheng X W, Luo A P, Zhao C J, Zhang H, Wen S $\mathrm{C}$, and $\mathrm{Xu} \mathrm{W} \mathrm{C,} \mathrm{"2} \mathrm{GHz} \mathrm{passively} \mathrm{harmonic} \mathrm{mode-locked} \mathrm{fiber} \mathrm{laser} \mathrm{by} \mathrm{a}$ microfiber-based topological insulator saturable absorber," Opt. Lett., vol. 38, no. 20, pp. 5212-5215 Dec. 2013.

[30] Koo J, Park J, Lee J, Jhon Y M, and Lee J H, "Femtosecond harmonic modelocking of a fiber laser at $3.27 \mathrm{GHz}$ using a bulk-like, MoSe2-based saturable absorber," Opt. Express, vol. 24, no. 19, pp. 10575 May. 2016.

[31] Rozhin A, Mou C, Zou C, Alaraimi M, Huang Q, and Wang T, "Passively harmonic mode-locked erbium-doped fiber laser at a $580 \mathrm{MHz}$ repetition rate based on carbon nanotubes film," Chin. Opt. Lett., vol. 16, no. 21, pp. 030019 Feb. 2018.

[32] Li C, Ma Y, Gao X, Niu F, Jiang T, Wang A, and Zhang Z, "1 GHz repetition rate femtosecond $\mathrm{Yb}$ : fiber laser for direct generation of carrier-envelope offset frequency," Appl. Opt., vol. 54, no. 10, pp. 8350-8353 Oct. 2015.

[33] Panasenko D, Polynkin P, Polynkin A, Moloney J V, Mansuripur M, and Peyghambarian $\mathrm{N}$, "Er-Yb femtosecond ring fiber oscillator with 1.1-W average power and GHz repetition rates," IEEE Photonics Technol. Lett., vol. 18, no. 23, pp. 853-855 Apr. 2006

[34] Amrani F, Haboucha A, Salhi M, Leblond H, Komarov A, Grelu P, and Sanchez F, "Passively mode-locked erbium-doped double-clad fiber laser operating at the 322nd harmonic," Opt. Lett., vol. 34, no. 22, pp. 2120-2122 Jul. 2009.

[35] Jun C S, Im J H, Yoo S H, Choi S Y, Rotermund F, Yeom D I, and Kim B $\mathrm{Y}$, "Low noise $\mathrm{GHz}$ passive harmonic mode-locking of soliton fiber laser using evanescent wave interaction with carbon nanotubes," Opt. Express, vol. 19, no. 25, pp. 19775-19780 Sep. 2011.

[36] Kim B Y, Chang S J, Yeom D I, Rotermund F, and Sun Y C, "Toward higher-order passive harmonic mode-locking of a soliton fiber laser," Opt. Lett., vol. 37, no. 24, pp. 1862 Jun. 2012.

[37] Tao S, Xu L, Chen G, Gu C, and Song H, "Ultra-High Repetition Rate Harmonic Mode-Locking Generated in a Dispersion and Nonlinearity Managed Fiber Laser," J. Lightwave Technol., vol. 34, no. 26, pp. 2354-2357 May.2016.

[38] Nelson L E, Jones D J, Tamura K, Haus H A, and Ippen E P, "Ultrashortpulse fiber ring lasers," Appl. Phys. B, vol. 65, no. 27, pp. 277-294 Apr. 1997.
[39] Tamura K, Nelson L, Haus H, and Ippen E, "Soliton versus nonsoliton operation of fiber ring lasers," Appl. Phys. Lett., vol. 64, no. 31, pp. 149-151 Nov. 1994.

[40] Nishizawa N, Nozaki Y, Itoga E, Kataura H, and Sakakibara Y, "Dispersion-managed, high-power, Er-doped ultrashort-pulse fiber laser using carbon-nanotube polyimide film," Opt. Express, vol. 19, no. 32, pp. 21874-21879 Oct. 2011.

[41] Mou C, Sergeyev S, Rozhin A, and Turistyn S, "All-fiber polarization locked vector soliton laser using carbon nanotubes," Opt. Lett., vol. 36, no. 11, pp. 3831-3833 Oct. 2011.

[42] Luo J L, Li L, and Ge Y Q, " L-band femtosecond fiber laser mode locked by nonlinear polarization rotation," IEEE Photon. Technol. Lett., vol. 26, no. 24, pp. 2438-2441 Dec. 2014

[43] Haus H A, Tamura K, Nelson L E, and Ippen E P, "Stretched-pulse additive pulse mode-locking in fiber ring lasers: theory and experiment," IEEE J. Quantum Electron., vol. 31, no. 33, pp. 591-598 Mar. 2002

[44] J. L. Wagener, D. G. Falquier, M. J. F. Digonnet, H. J. Shaw, and L. Fellow, "A Mueller Matrix Formalism for Modeling Polarization Effects in ErbiumDoped Fiber," J. Lightwave Technol., vol. 16, no. 10, pp. 1942-1943, Feb. 1998.

[45] D. W. Hall and M. J. Weber, "Spectral and polarization hole burning in Nd:glass lasers," IEEE J. Quantum Electron., vol. 19, no. 11, pp. 1704-1717, Jan. 2003.

[46] H. Zhang, D. Y. Tang, X. Wu, and L. M. Zhao, "Multi-wavelength dissipative soliton operation of an erbium-doped fiber laser," Opt. Express, vol. 17, no. 15, pp. 12692-7, Jul. 2009.

[47] Zhang H, Tang D, Knize R J, Zhao L, Bao Q, and Loh K P, "Graphene mode locked, wavelength-tunable, dissipative soliton fiber laser," Appl. Phys. Lett., vol. 96, no. 34, pp. 51 Mar. 2010.

[48] H. Shisheng, W. Yonggang, Y. Peiguang, Z. Junqing, L. Huiquan, and L. Rongyong, "Tunable and switchable multi-wavelength dissipative soliton generation in a graphene oxide mode-locked Yb-doped fiber laser," Opt. Express, vol. 22, no. 10, pp. 11417-11426, May. 2014.

[49] Cheng K N, Lin Y H, Yamashita S, and Lin G R, "Harmonic OrderDependent Pulsewidth Shortening of a Passively Mode-Locked Fiber Laser With a Carbon Nanotube Saturable Absorber," IEEE Photonics J., vol. 4, no. 17, pp. 1542-1552 Oct. 2012.

[50] Renninger W H, Chong A, and Wise F W, "Area theorem and energy quantization for dissipative optical solitons," JOSA B, vol. 27, no. 35, pp. 1978-1982 Sep. 2010.

[51] Eberly J, "Area Theorem rederived," Opt. Express, vol. 2, no. 36, pp. 173 176 Mar. 1998.

Qianqian Huang was born in Wenzhou, Zhejiang, China, in 1993. She received the B.S. degree from Zhejiang Science and Technology University, Zhejiang, China, in 2016. And she is currently working towards the M. Eng. degree at the Key Lab of Specialty Fiber Optics and Optical Access Network, Shanghai University, Shanghai, in 2016. Her current research focus is on high repetition rate fiber laser and nonlinear fiber optics.

Chuanhang Zou was born in Hangzhou, Zhejiang, China, in 1992. He received the B.S. degree from Tianjin University of Technology, Tianjin, China, in 2016, the M. Eng. Degree at the Key Lab of Specialty Fiber Optics and Optical Access Network, Shanghai University, Shanghai, in 2019. 
Tianxing Wang was born on 1992 in Jiangsu, China. He received the M. S. degree in communication \& information system from Shanghai University, Shanghai, in 2018. He joined Key Laboratory of Materials for High Power Laser, Shanghai Institute of Optics and Fine Mechanics, Shanghai, China, in 2018. He has been involved extensively in the ultrafast fiber laser research activities in the lab. His research interests include mode-locked fiber lasers, large energy fiber lasers, and gain switched lasers.

\begin{abstract}
Aleksey Rozhin received the M.S. degree in physics from Taras Shevchenko National University of Kiev, Kiev, Ukraine, in 1998, and the Ph.D. degree in solid state physics from the Institute for Semiconductor Physics, National Academy of Science of Ukraine, Kiev, Ukraine, in 2001. From 2001 to 2002, he was a Researcher with the Institute for Semiconductor Physics NASU, Ukraine. From 2002 to 2005, he was JSPS, AIST Postdoctoral Fellow with the National Institute of Advanced Industrial Science and Technology, Japan. From 2005 to 2009, he was a Senior Research Associate with the Centre for Advanced Photonics and Electronics, University of Cambridge, U.K. Since 2009, he has been with Aston University, Birmingham, U.K., first as a Lecturer, where he became a Senior Lecturer in 2012, in Nanotechnology. His current research interests include nanomaterials synthesis, nanomaterials surface functionalization, development of nanocomposites for photonic applications, solid-state physics, and biophysics. He is working on designing and developing novel ultrafast lasers, polymer waveguides, optical switches, signal regenerative filters, fibre and waveguide sensors, microfluidic devices. He has published more than 60 journal and ten conference papers in the area of photonics and nonlinear optical materials, fibre lasers, fibre communication devices, and filed ten patent applications.
\end{abstract}

Chengbo Mou received the B.Eng. degree in electronic science and technologies from Tianjin University, Tianjin, China, in 2004, the M.Sc. degree in photonics and optoelectronic devices from the University of St Andrews, St Andrews, U.K., in 2005, the Ph.D. degree in photonics from the Aston Institute of Photonic Technologies, Aston University, Birmingham, U.K., in 2012. He then worked as an Industrial Research Fellow with Aston University. In 2016, he joined in the Key Laboratory of Specialty Fiber Optics and Optical Access Networks as a Full Professor. His research interests include nanophotonics, nanomaterial-based nonlinear photonic devices, ultrafast fiber lasers, novel type of mode locked lasers, and nonlinear applications of advanced fiber grating devices. $\mathrm{He}$ is the recipient of National "Young 1000 Talent" programme of China, the Young Eastern Scholar Fellowship from the Shanghai Institute of Higher Learning. 